CERN-TH.6336/91

IFUM $412 / \mathrm{FT}$

hepth@xxx/9202069

\title{
QUANTUM CONSERVED CURRENTS IN AFFINE TODA THEORIES
}

\author{
G.W. DELIUS and M.T. GRISARUF \\ Theory Division, CERN, 1211 Geneva 23, Switzerland \\ and \\ D. ZANON \\ Dipartimento di Fisica dell' Università di Milano and \\ INFN, Sezione di Milano, I-20133 Milano, Italy
}

\begin{abstract}
We study the renormalization and conservation at the quantum level of higher-spin currents in affine Toda theories with particular emphasis on the nonsimply-laced cases. For specific examples, namely the spin-3 current for the $a_{3}^{(2)}$ and $c_{2}^{(1)}$ theories, we prove conservation to all-loop order, thus establishing the existence of factorized S-matrices. For these theories, as well as the simply-laced $a_{2}^{(1)}$ theory, we compute one-loop corrections to the corresponding higher-spin charges and study charge conservation for the three-particle vertex function. For the $a_{3}^{(2)}$ theory we show that although the current is conserved, anomalous threshold singularities spoil the conservation of the corresponding charge for the on-shell vertex function, implying a breakdown of some of the bootstrap procedures commonly used in determining the exact S-matrix.
\end{abstract}

CERN-TH.6336/91

IFUM $412 / \mathrm{TH}$

December 1991

*On leave from Brandeis University, Waltham, MA 02254, USA

Work partially supported by the National Science Foundation under grant PHY-88-18853 and by INFN 


\section{Introduction}

Affine Toda field theories describe a class of massive systems which are classically integrable; from the identification of the field equations as compatibility conditions for a Lax pair follows the existence of an infinite number of classically conserved higher-spin currents $J_{+}^{(s)}, J_{-}^{(s)}[$ [ $]$

$$
\partial_{-} J_{+}^{(s)}+\partial_{+} J_{-}^{(s)}=0
$$

Unless the conservation is spoiled by quantum anomalies, the existence of the corresponding charges $Q^{(s)}$ implies the factorization and elasticity of the S-matrix [2, 3]. Unitarity and a bootstrap principle lead then to its exact determination.

The quantum-conservation of the higher-spin currents has been investigated by a number of authors for the simplest of the affine Toda theories, namely the sine(or sinh-) Gordon system, as well as its supersymmetric counterpart [4, 5. By using BPHZ techniques, or conformal field theory methods, it has been shown that renormalized currents can be defined which indeed are conserved. Similar investigations have been carried out in the context of perturbed conformal field theories [6].

Our interest in the quantum-conservation arose out of work on exact S-matrices for nonsimply-laced affine Toda theories. For simply-laced affine Toda theories exact S-matrices were constructed some time ago [7, \&] but similar constructions had failed for the nonsimply-laced cases, suggesting that perhaps the classical integrability breaks down at the quantum level. It was the realization that higher-spin quantum-conserved currents exist that gave the impetus for a new, successful effort, to determine hitherto unknown S-matrices for these systems [9]. In this paper we present details of the construction of such currents and of corresponding charges. We also discuss the issue of charge conservation in the presence of anomalous threshold singularities, and implications for the bootstrap program of the S-matrix.

Because explicit formulas are not known for currents of high spin and also to avoid algebraic complexity, we have restricted ourselves to the lowest nontrivial spin $s \geq 2$ ( $\operatorname{spin} s=1$ corresponds to the stress tensor), for nonsimply-laced Toda theories with only two bosonic fields, namely the spin-3 current for the $a_{3}^{(2)}$ and $c_{2}^{(1)}$ theories. However, to set the notation and for comparison, we also consider the spin-2 current for the $a_{2}^{(1)}$ simply-laced theory (the results are easily extended to the simply-laced $a_{n}^{(1)}$ case for any $n$ ). In general, two-dimensional power counting arguments and Lorentz invariance suggest that any violation at the quantum level of the classical conservation laws for a spin $s$ current can occur at most at $l=s-1$ loops, and from a restricted class of diagrams; for bosonic theories they are diagrams obtained by Wick-contracting the currents with just one factor of the interaction lagrangian. We are thus able to obtain all-loop results for the absence of anomalies in the currents.

The affine Toda theories have interaction lagrangians which are exponentials of sums of fields. From these one can separate the quadratic parts which correspond to mass terms. We have studied the conservation of currents by using two different methods: massive perturbation theory and BPHZ techniques, and massless 
perturbation theory which treats the whole exponentials as interaction terms and is essentially equivalent to OPE techniques. Both methods lead to the same conclusions, but calculations using massless perturbation methods are much simpler beyond one loop and are the ones that will be presented here.

Our paper is organized as follows: in Section 2 we set up the notation and describe the general procedure for constructing quantum-conserved currents. In Section 3 we carry out the construction for the cases mentioned above; the exact currents we obtain consist of classical parts, plus quantum corrections. In Section 4 we define, and compute up to one-loop order the corresponding charges. In Section 5 we show that the one-loop corrections to the classical charges are consistent with quantum corrections to the masses of the particles, by studying the chargeconservation relations for the three-point on-shell vertex functions. In Section 6 we show how these relations are affected by the presence of anomalous threshold singularities, and discuss implications for the S-matrix bootstrap principle. Section 7 contains discussion and conclusions.

\section{Massless perturbation theory conventions}

We consider lagrangians of the form

$$
\beta^{2} \mathcal{L}=-\frac{1}{2} \vec{\phi} \square \vec{\phi}-\mu^{2} \sum_{i} q_{i} e^{\vec{\alpha}_{i} \cdot \vec{\phi}}
$$

Here the $\vec{\alpha}_{i}$ are the positive simple roots of a rank $n$ Lie algebra augmented by (the negative of ) a maximal root and $\vec{\phi}=\left(\phi_{1}, \phi_{2}, \ldots \phi_{n}\right)$ are bosonic fields describing $n$ massive particles. The quantum lagrangian is defined by normal-ordering the exponentials after which all Green's functions of the basic fields are free of any divergences. The Kač labels $q_{i}$ are chosen so that the one-point functions vanish. $\beta$ is the coupling constant, and $\mu$ sets the mass scale; we choose $\mu=1$. At the classical level these theories possess conserved currents of spins $s$ equal to the exponents of the algebra modulo the Coxeter number [1].

We work in Minkowski space and use light-cone coordinates; for convenience, whenever possible, we use a notation reminiscent of Euclidean space

$$
\begin{aligned}
z \equiv x^{+} & =\frac{1}{\sqrt{2}}\left(x^{0}+x^{1}\right) & & \bar{z} \equiv x^{-}=\frac{1}{\sqrt{2}}\left(x^{0}-x^{1}\right) \\
\partial \equiv \partial_{+} & =\frac{1}{\sqrt{2}}\left(\partial_{0}+\partial_{1}\right) & & \bar{\partial} \equiv \partial_{-}=\frac{1}{\sqrt{2}}\left(\partial_{0}-\partial_{1}\right) \\
\square & =2 \partial \bar{\partial} & &
\end{aligned}
$$

and also

$$
J^{(s)} \equiv J_{+}^{(s)}=\frac{1}{\sqrt{2}}\left(J_{0}^{(s)}+J_{1}^{(s)}\right) \quad, \quad \bar{J}^{(s)} \equiv J_{-}^{(s)}=\frac{1}{\sqrt{2}}\left(J_{0}^{(s)}-J_{1}^{(s)}\right)
$$

We perform calculations in $x$-space with massless bosonic propagators

$$
\langle\phi(z, \bar{z}) \phi(0,0)\rangle=-\frac{\beta^{2}}{4 \pi} \log (2 z \bar{z})
$$


We consider currents of the form

$$
\begin{aligned}
J^{(s)} & =\sum a_{p q} \partial^{p_{1}} \phi_{q_{1}} \cdots \partial^{p_{n}} \phi_{q_{n}} \\
\bar{J}^{(s)} & =\sum_{i}\left(\sum b_{i t r} \partial^{t_{1}} \phi_{r_{1}} \cdots \partial^{t_{n}} \phi_{r_{n}}\right) e^{\vec{\alpha}_{\cdot} \cdot \vec{\phi}}
\end{aligned}
$$

with $\sum p_{i}=s+1, \sum t_{i}=s-1$ and with coefficients $a_{p q}=a_{p q}^{(0)}+\beta^{2} a_{p q}^{(1)}+\cdots$, etc.. The coefficients $a_{p q}^{(0)}$ and $b_{i t r}^{(0)}$ are determined (up to the shift $J \rightarrow J+\partial \Lambda, \bar{J} \rightarrow \bar{J}-\bar{\partial} \Lambda$ ) by the classical conservation laws $\bar{\partial} J^{(s)}+\partial \bar{J}^{(s)}=0$. At the quantum level we compute

$$
\bar{\partial}_{z}\langle J(z, \bar{z})\rangle \equiv \bar{\partial}_{z}\left\langle J(z, \bar{z}) \exp \left(i \int d^{2} w \mathcal{L}_{\text {int }}(w, \bar{w})\right)\right\rangle_{0}
$$

Potential anomalies would correspond to local contributions which cannot be written as the $\partial$-derivative of some suitable expression. We attempt to determine the coefficients $a_{p q}$ to cancel such contributions.

For the lagrangian in eq. (2.1) we need only consider expanding the exponential in eq. (2.6) to first order in $\mathcal{L}_{\text {int }}$. Since the component $J^{(s)}$ of the currents contains exclusively terms of the form $\partial^{p} \phi$ the Wick contractions in the above matrix element will lead to a sum of terms of the form

$$
\int d^{2} w A(z, \bar{z}) \frac{1}{(z-w)^{n}} B(w, \bar{w})
$$

where $A, B$, are products of fields and their $\partial$ derivatives.

Since any potential anomalies are local, the $\bar{\partial}$-derivative in eq. (2.6) must act on the $(z-w)^{-n}$ factor. We use the standard prescription

$$
\bar{\partial}_{z} \frac{1}{(z-w)^{n}}=\frac{2 \pi i}{(n-1) !} \partial_{w}^{n-1} \delta^{(2)}(z-w)
$$

so that we are led to consider a sum of terms of the form

$$
A(z, \bar{z}) \partial^{n-1} B(z, \bar{z})
$$

that we should be able to rewrite as some $\partial \bar{J}(z, \bar{z})$. We are not interested in the actual form of $\bar{J}$, and so in our calculations we shall freely "integrate by parts" on $z$, i.e. drop total $\partial$ derivatives.

A spin $s$ current contains at most $s+1$ factors of fields. Therefore the Wick contractions lead to contributions only up to $s$ loops. However, the $s$-loop contribution is automatically a total derivative so need not be considered further. Thus, for example,

$$
\begin{aligned}
& \bar{\partial}_{z}\left\langle(\partial \phi)^{4}\left(-\frac{i}{\beta^{2}} \int d^{2} w e^{\phi}\right)\right\rangle \\
& \quad=-i \bar{\partial}_{z} \int d^{2} w\left[4(\partial \phi)^{3} \frac{-1}{4 \pi(z-w)}+6(\partial \phi)^{2} \frac{\beta^{2}}{16 \pi^{2}(z-w)^{2}}\right.
\end{aligned}
$$




$$
\begin{gathered}
\left.+4 \partial \phi \frac{-\beta^{4}}{64 \pi^{3}(z-w)^{3}}+\frac{\beta^{6}}{256 \pi^{4}(z-w)^{4}}\right] e^{\phi} \\
\leadsto-2(\partial \phi)^{3} e^{\phi}-\frac{3 \beta^{2}}{4 \pi}(\partial \phi)^{2} \partial e^{\phi}-\frac{\beta^{4}}{16 \pi^{2}} \partial \phi \partial^{2} e^{\phi}-\frac{\beta^{6}}{768 \pi^{3}} \partial^{3} e^{\phi} \\
\leadsto\left[-4-\frac{3 \beta^{2}}{2 \pi}+\frac{\beta^{4}}{16 \pi^{2}}\right] \partial^{3} \phi e^{\phi}
\end{gathered}
$$

where we have dropped the $\beta^{6}$ contribution which is manifestly a total derivative, and also used the identities (which are valid up to total $\partial$ derivatives)

$$
\begin{aligned}
\partial\left(\partial^{2} \phi e^{\phi}\right) & =\left(\partial^{3} \phi+\partial^{2} \phi \partial \phi\right) e^{\phi} \sim 0 \\
\partial\left((\partial \phi)^{2} e^{\phi}\right) & =\left(2 \partial \phi \partial^{2} \phi+(\partial \phi)^{3}\right) e^{\phi} \sim 0
\end{aligned}
$$

\section{Quantum-conserved currents}

In this section we construct quantum-conserved currents for the $a_{2}^{(1)}$ simply-laced affine Toda theory, and for the nonsimply-laced $a_{3}^{(2)}$ and $c_{2}^{(1)}$ theories. These are theories with two bosonic fields, general enough to illustrate the issues we wish to discuss. All these theories have a conserved spin $s=1$ current, namely the stresstensor $T \equiv T_{++}, \bar{T} \equiv T_{+-}$, satisfying $\bar{\partial} T+\partial \bar{T}=0$.

\subsection{The $a_{2}^{(1)}$ affine Toda theory}

The lagrangian for this simply-laced theory is given by

$$
\begin{aligned}
\beta^{2} \mathcal{L}= & -\frac{1}{2} \phi_{1} \square \phi_{1}-\frac{1}{2} \phi_{2} \square \phi_{2}-e^{\sqrt{2} \phi_{1}} \\
& -e^{-\frac{1}{\sqrt{2}} \phi_{1}-\sqrt{\frac{3}{2}} \phi_{2}}-e^{-\frac{1}{\sqrt{2}} \phi_{1}+\sqrt{\frac{3}{2}} \phi_{2}}
\end{aligned}
$$

General considerations [1] establish the existence of a classically conserved spin-2 current.

We consider, based on the $\phi_{2} \rightarrow-\phi_{2}$ symmetry of the lagrangian,

$$
J^{(2)}=A\left(\partial \phi_{1}\right)^{2} \partial \phi_{2}+B\left(\partial \phi_{2}\right)^{3}+C \partial \phi_{1} \partial^{2} \phi_{2}
$$

Wick-contracting with the interaction lagrangian we obtain $\left(\alpha \equiv \frac{\beta^{2}}{2 \pi}\right)$

$$
\begin{gathered}
\bar{\partial}\left\langle J^{(2)}\left(-\frac{i}{\beta^{2}} \int d^{2} w e^{\sqrt{2} \phi_{1}}\right)\right\rangle \sim\left[\left(1+\frac{\alpha}{2}\right) A-\frac{C}{\sqrt{2}}\right] \partial^{2} \phi_{2} e^{\sqrt{2} \phi_{1}} \\
\bar{\partial}\left\langle J^{(2)}\left(-\frac{i}{\beta^{2}} \int d^{2} w e^{-\frac{1}{\sqrt{2}} \phi_{1}-\sqrt{\frac{3}{2}} \phi_{2}}\right)\right\rangle \\
\sim\left[\frac{\sqrt{3}}{2}\left(\left(1+\frac{\alpha}{2}\right) A-\frac{C}{\sqrt{2}}\right) \partial^{2} \phi_{1}+\left(\frac{\alpha}{8} A+\left(\frac{3}{2}+\frac{9}{8} \alpha\right) B+\frac{1}{2 \sqrt{2}} C\right) \partial^{2} \phi_{2}\right. \\
\left.-\frac{1}{2 \sqrt{2}}(A+3 B) \partial \phi_{1} \partial \phi_{2}\right] e^{-\frac{1}{\sqrt{2}} \phi_{1}-\sqrt{\frac{3}{2}} \phi_{2}}
\end{gathered}
$$


where we have kept only the local part of the left-hand-side, as in eq. (2.10). We do not need to calculate separately contractions with the third exponential in the lagrangian because of the symmetry under $\phi_{2} \rightarrow-\phi_{2}$. We have made use of identities (up to total $\partial$ derivatives), similar to the ones in eq. (2.11).

Current conservation requires that the terms on the right-hand-sides in eq. (3.3), which are not total $\partial$ derivatives of some $\bar{J}^{(s)}$, vanish:

$$
\begin{aligned}
& \left(1+\frac{\alpha}{2}\right) A-\frac{1}{\sqrt{2}} C=0 \\
& \frac{\alpha}{8} A+\left(\frac{3}{2}+\frac{9}{8} \alpha\right) B+\frac{1}{2 \sqrt{2}} C=0 \\
& A+3 B=0
\end{aligned}
$$

These three homogeneous equations with three unknowns can be satisfied nontrivially since the determinant of the system vanishes and thus we find, up to an overall normalization factor, a quantum-conserved spin-2 current

$$
J^{(2)}=\left(\partial \phi_{1}\right)^{2} \partial \phi_{2}-\frac{1}{3}\left(\partial \phi_{2}\right)^{3}+\sqrt{2}\left(1+\frac{\beta^{2}}{4 \pi}\right) \partial \phi_{1} \partial^{2} \phi_{2}
$$

The terms independent of $\beta^{2}$ give of course the $J$ component of the classical conserved current.

We emphasize that it was not obvious a priori that the equations for the coefficients $A, B, C$ would have a nontrivial solution so that a quantum-conserved current exists. However this result was known, and also expected since S-matrices for the $a_{n}^{(1)}$ series had been constructed [7], implying the existence of higher-spin quantumconserved charges. In fact the result in eq. (3.5) can be generalized to the $a_{n}^{(1)}$ theories for any $n$ : using the same techniques it is easy to show that the current

$$
J^{(2)}=K_{a b c} \partial \phi_{a} \partial \phi_{b} \partial \phi_{c}+K_{a b} \partial \phi_{a} \partial^{2} \phi_{b}
$$

is quantum-conserved provided the coefficients satisfy

$$
\left(1+\frac{\beta^{2}}{4 \pi}\right) K_{a b c} \alpha_{a}^{i}=\frac{1}{6}\left(K_{a b} \alpha_{a}^{i} \alpha_{c}^{i}+K_{a c} \alpha_{a}^{i} \alpha_{b}^{i}\right)
$$

where $\vec{\alpha}^{i}$ are the roots of the $a_{n}^{(1)}$ theory normalized to $\left|\vec{\alpha}^{i}\right|^{2}=2$. Since at the classical level a spin-2 conserved current is known to exist [1], the above equations have a solution if one drops the $\beta^{2}$ term, with some coefficients $K_{a b c}^{(0)}, K_{a b}^{(0)}$. It is obvious then that the quantum current also exists, with $K_{a b c}=K_{a b c}^{(0)}, K_{a b}=\left(1+\frac{\beta^{2}}{4 \pi}\right) K_{a b}^{(0)}$. We note that the result in eq. (3.7), with the common factor $1+\frac{\beta^{2}}{4 \pi}$ for any $i$, holds only because $a_{n}^{(1)}$ is simply-laced.

\subsection{The $a_{3}^{(2)}$ affine Toda theory}

For this nonsimply-laced theory the lagrangian is

$$
\beta^{2} \mathcal{L}=-\frac{1}{2} \phi_{1} \square \phi_{1}-\frac{1}{2} \phi_{2} \square \phi_{2}-e^{-\phi_{1}-\phi_{2}}-e^{-\phi_{1}+\phi_{2}}-e^{2 \phi_{1}}
$$


It has a spin-3 classically conserved current [1], so we start (using also the symmetry of the lagrangian under $\phi_{2} \rightarrow-\phi_{2}$ ) with

$$
J^{(3)}=A\left(\partial \phi_{1}\right)^{2}\left(\partial \phi_{2}\right)^{2}+B\left(\partial \phi_{1}\right)^{4}+C\left(\partial \phi_{2}\right)^{4}+D \partial \phi_{1} \partial \phi_{2} \partial^{2} \phi_{2}+E\left(\partial^{2} \phi_{1}\right)^{2}+F\left(\partial^{2} \phi_{2}\right)^{2}
$$
tives)

We find, writing the results in terms of an independent set (up to total $\partial$ deriva-

$$
\begin{aligned}
& \bar{\partial}\left\langle J^{(3)}\left(-\frac{i}{\beta^{2}} \int d^{2} w e^{2 \phi_{1}}\right)\right\rangle \\
& \sim\left([2(1+\alpha) A-D] \partial^{2} \phi_{2} \partial \phi_{2}-\left[2\left(1+3 \alpha+\alpha^{2}\right) B-2 E\right] \partial^{3} \phi_{1}\right) e^{2 \phi_{1}} \\
& \bar{\partial}\left\langle J^{(3)}\left(-\frac{i}{\beta^{2}} \int d^{2} w e^{-\phi_{1}-\phi_{2}}\right)\right\rangle \\
& \quad \leadsto\left(\left[\left(1+\frac{\alpha}{2}\right) A-(6+3 \alpha) B-\frac{1}{2} D\right] \partial^{2} \phi_{1} \partial \phi_{2}\right. \\
& \quad+\left[\left(1+\frac{\alpha}{2}\right) A-2 B-(4+3 \alpha) C-\frac{1}{2} D\right] \partial^{2} \phi_{2} \partial \phi_{1} \\
& \quad+\left[\left(\frac{\alpha}{2}+\frac{\alpha^{2}}{8}\right) A+\left(4+3 \alpha+\frac{\alpha^{2}}{4}\right) B-\frac{\alpha}{8} D-E\right] \partial^{3} \phi_{1} \\
& \quad+\left[\left(\frac{\alpha}{2}+\frac{\alpha^{2}}{8}\right) A+\left(4+3 \alpha+\frac{\alpha^{2}}{4}\right) C+\left(\frac{1}{2}+\frac{\alpha}{8}\right) D-F\right] \partial^{3} \phi_{2} \\
& \left.\quad+[2(B-C)]\left(\partial \phi_{2}\right)^{2} \partial \phi_{1}\right) e^{-\phi_{1}-\phi_{2}}
\end{aligned}
$$

Again, the remaining exponential need not be considered because of the $\phi_{2} \rightarrow-\phi_{2}$ symmetry.

Requiring that the right hand side vanish we obtain a set of equations for the coefficients $A, B, \ldots . F$, which have a nontrivial solution leading to the quantumconserved current

$$
\begin{aligned}
J^{(3)}= & \left(1+\frac{\beta^{2}}{4 \pi}\right)\left(\partial \phi_{1}\right)^{2}\left(\partial \phi_{2}\right)^{2}-\frac{\beta^{2}}{24 \pi}\left(\partial \phi_{1}\right)^{4}-\frac{\beta^{2}}{24 \pi}\left(\partial \phi_{2}\right)^{4} \\
& +\left(2+\frac{3 \beta^{2}}{2 \pi}+\frac{\beta^{4}}{4 \pi^{2}}\right) \partial \phi_{1} \partial \phi_{2} \partial^{2} \phi_{2}-\frac{\beta^{2}}{24 \pi}\left(1+\frac{3 \beta^{2}}{2 \pi}+\frac{\beta^{4}}{4 \pi^{2}}\right)\left(\partial^{2} \phi_{1}\right)^{2} \\
& +\left(1+\frac{23 \beta^{2}}{24 \pi}+\frac{\beta^{4}}{4 \pi^{2}}+\frac{\beta^{6}}{48 \pi^{3}}\right)\left(\partial^{2} \phi_{2}\right)^{2}
\end{aligned}
$$

We note that in addition to the renormalization of terms already present in the classical current new terms are generated at the quantum level. In particular, the appearance of the $\left(\partial^{2} \phi_{1}\right)^{2}$ term has important consequences that will be discussed later on.

The existence of the quantum-conserved current $J^{(3)}$ implies that this theory must have factorizable, elastic S-matrices. 


\subsection{The $c_{2}^{(1)}$ affine Toda theory}

Again this is a nonsimply-laced theory, with the lagrangian given by

$$
\beta^{2} \mathcal{L}=-\frac{1}{2} \phi_{1} \square \phi_{1}-\frac{1}{2} \phi_{2} \square \phi_{2}-e^{\sqrt{2}\left(\phi_{1}-\phi_{2}\right)}-e^{-\sqrt{2}\left(\phi_{1}+\phi_{2}\right)}-2 e^{\sqrt{2} \phi_{2}}
$$

We consider the spin-3 current, even in $\phi_{1}$,

$$
J^{(3)}=A\left(\partial \phi_{1}\right)^{4}+B\left(\partial \phi_{2}\right)^{4}+C\left(\partial \phi_{1}\right)^{2}\left(\partial \phi_{2}\right)^{2}+D\left(\partial \phi_{1}\right)^{2} \partial^{2} \phi_{2}+E\left(\partial^{2} \phi_{1}\right)^{2}+F\left(\partial^{2} \phi_{2}\right)^{2}
$$

We obtain

$$
\begin{aligned}
& \bar{\partial}\left\langle J^{(3)}\left(-\frac{2 i}{\beta^{2}} \int d^{2} w e^{\sqrt{2} \phi_{2}}\right)\right\rangle \\
& \leadsto\left(\left[-\sqrt{2}\left(4+6 \alpha+\alpha^{2}\right) B+2 \sqrt{2} F\right] \partial^{3} \phi_{2}+[(4+2 \alpha) C+2 \sqrt{2} D] \partial^{2} \phi_{1} \partial \phi_{1}\right) e^{\sqrt{2} \phi_{2}} \\
& \bar{\partial}\left\langle J^{(3)}\left(-\frac{i}{\beta^{2}} \int d^{2} w e^{\sqrt{2}\left(\phi_{1}-\phi_{2}\right.}\right)\right\rangle \\
& \sim\left([(6+6 \alpha) A-(1+\alpha) C-\sqrt{2} D] \partial^{2} \phi_{1} \partial \phi_{1}\right. \\
& +[2 A+(4+6 \alpha) B-(1+\alpha) C-\sqrt{2} D] \partial^{2} \phi_{2} \partial \phi_{2} \\
& +\left[\left(\sqrt{2}-\frac{\alpha^{2}}{\sqrt{2}}\right) A-\left(\frac{1}{\sqrt{2}}+\sqrt{2} \alpha+\frac{\alpha^{2}}{2 \sqrt{2}}\right) C+\frac{\alpha}{2} D+\sqrt{2} E\right] \partial^{3} \phi_{1} \\
& +\left[-\sqrt{2} A+\frac{\alpha^{2}}{\sqrt{2}} B+\left(\frac{1}{\sqrt{2}}+\sqrt{2} \alpha+\frac{\alpha^{2}}{2 \sqrt{2}}\right) C+\left(1+\frac{\alpha}{2}\right) D-\sqrt{2} F\right] \partial^{3} \phi_{2} \\
& \left.\quad-2 \sqrt{2}(A-B)\left(\partial \phi_{2}\right)^{2} \partial \phi_{1}\right) e^{\sqrt{2}\left(\phi_{1}-\phi_{2}\right)}
\end{aligned}
$$

where we have used again various identities that follow from integration by parts. We

obtain equations which have a nontrivial solution leading to the quantum-conserved current

$$
\begin{aligned}
J^{(3)=} & \left(\partial \phi_{1}\right)^{4}+\left(\partial \phi_{2}\right)^{4}-6\left(1+\frac{\beta^{2}}{2 \pi}\right)\left(\partial \phi_{1}\right)^{2}\left(\partial \phi_{2}\right)^{2}+\left(2+\frac{3 \beta^{2}}{2 \pi}+\frac{\beta^{4}}{8 \pi^{2}}\right)\left(\partial^{2} \phi_{2}\right)^{2} \\
& +6\left(\sqrt{2}+\frac{3 \beta^{2}}{2 \sqrt{2} \pi}+\frac{\beta^{4}}{4 \sqrt{2} \pi^{2}}\right)\left(\partial \phi_{1}\right)^{2} \partial^{2} \phi_{2}-\left(4+\frac{6 \beta^{2}}{\pi}+\frac{23 \beta^{4}}{8 \pi^{2}}+\frac{3 \beta^{6}}{8 \pi^{3}}\right)\left(\partial^{2} \phi_{1}\right)^{2}
\end{aligned}
$$

\section{One-loop charges}

From the quantum-conserved currents we can construct corresponding higher-spin charges. Their existence has strong implications for the S-matrices of the massive Toda theories we are considering: the $n$-body S-matrices factorize into products of 2-body elastic S-matrices. Furthermore, the S-matrix bootstrap principle [2] implies certain relations between the charges of the particles participating in the bootstrap 
[10, 8]. Conversely, the violation of these relations, which can be examined once the charges are given, implies a breakdown of the bootstrap. These are some of the issues we wish to discuss in the rest of this article. Since we are now interested in on-shell properties of the theories, we will do our calculations in terms of massive states and propagators, with the classical masses and the interactions we shall need obtained from the quadratic and higher-order parts of the exponentials in the Toda lagrangians.

From the current conservation law follows the time-independence of the corresponding charge operators $Q^{(s)}=\int d x^{+} J_{+}^{(s)}$, hence $Q^{(s)}$ commutes with the Hamiltonian of the system. Single particle states are therefore eigenstates of the charge operators. Since the charge $Q^{(s)}$ has helicity $s$, its action on single-particle states is

$$
Q^{(s)}|p\rangle=\beta^{2} \omega p_{+}^{s}|p\rangle
$$

(The $\beta^{2}$ factor has been introduced to account for our normalization of the fields [1], cf. the $\beta^{2}$ in the propagator, eq. (2.4).) Acting on a product of wave-packets, the operator $\exp i Q^{(s)}, s>1$, displaces them relative to each other, so that, since $Q^{(s)}$ commutes with the S-operator, a multiparticle scattering amplitude is equal to one where well-separated wave-packets scatter pairwise, i.e. the S-matrix factorizes into products of two-body S-matrices. The elasticity is based on the charge conservation relation for the process $p_{a}+p_{b}+\ldots \rightarrow p_{f}+p_{g}+\ldots$

$$
\omega_{a} p_{+a}^{s}+\omega_{b} p_{+b}^{s}+\ldots=\omega_{f} p_{+f}^{s}+\omega_{g} p_{+g}^{s}+\ldots
$$

which can be satisfied, generically, only if the outgoing momenta are at most a permutation of the incoming momenta.

We will refer to $\omega$ as the particle charge and compute it in terms of on-shell matrix elements $\left\langle p\left|J^{(s)}(0)\right| p\right\rangle \sim p_{+}^{s+1}$, by

$$
\left\langle q\left|Q^{(s)}\right| p\right\rangle=\left\langle q\left|\int d x^{+} J_{+}^{(s)}(x)\right| p\right\rangle=2 \pi \delta\left(p_{+}-q_{+}\right)\left\langle p\left|J^{(s)}(0)\right| p\right\rangle
$$

We are using a normalization

$$
\langle q \mid p\rangle=\delta\left(p_{1}-q_{1}\right)=\frac{p_{+}}{p_{0}} \delta\left(p_{+}-q_{+}\right)
$$

We obtain

$$
\omega=\frac{2 \pi p_{0}}{\beta^{2} p_{+}^{s+1}}\left\langle p\left|J^{(s)}(0)\right| p\right\rangle
$$

At the classical level, for the cases discussed in the previous sections, the charges can be read off from the quadratic parts of the classical currents. For the $a_{2}^{(1)}$ theory we observe that $m_{1}=m_{2}$ and the charge is not diagonal in the $\phi_{1}, \phi_{2}$ basis. However $\frac{1}{\sqrt{2}}\left(\phi_{1} \mp i \phi_{2}\right)$ are the proper combinations which diagonalize it and we have, for the spin-2 charge

$$
\omega_{ \pm}^{(0)}= \pm \frac{1}{\sqrt{2}}
$$


Similarly, in the $a_{3}^{(2)}$ theory, from the $O\left(\beta^{0}\right)$ quadratic part of the current, we have

$$
\omega_{1}^{(0)}=0 \quad, \quad \omega_{2}^{(0)}=1
$$

Finally, in the $c_{2}^{(1)}$ theory,

$$
\omega_{1}^{(0)}=-4 \quad, \quad \omega_{2}^{(0)}=2
$$

At the quantum level we write $\omega=\omega^{(0)}+\beta^{2} \omega^{(1)}+\cdots$. In the following subsections we will compute the one-loop contributions to these charges. These arise from three sources: one-loop contributions from the classical parts of the currents, tree level contributions from the $O\left(\beta^{2}\right)$ parts of the currents, and wave-function renormalization contributions.

\subsection{The $a_{2}^{(1)}$ theory}

From eq. (3.1) we read the masses and the cubic terms in the interaction lagrangian

$$
\begin{aligned}
& m_{1}^{2}=m_{2}^{2}=3 \\
& \beta^{2} \mathcal{L}^{(3)}=-\frac{\sqrt{2}}{4} \phi_{1}^{3}+\frac{3 \sqrt{2}}{4} \phi_{1} \phi_{2}^{2}
\end{aligned}
$$

We compute up to $O\left(\beta^{2}\right)$ contributions to $\frac{2 \pi p_{0}}{\beta^{2} p_{+}^{3}}\left\langle 1\left|J^{(2)}\right| 2\right\rangle$, with $J^{(2)}$ given in eq. (3.5). They correspond to the Feynman diagrams represented in Fig.1 and are given by

$$
\begin{gathered}
\text { (a) : } \frac{3 \sqrt{2} \beta^{2}}{p_{+}^{3}(2 \pi)^{2}} \int d^{2} k \frac{k_{+}(p-k)_{+} p_{+}}{\left[k^{2}-3\right]\left[(k-p)^{2}-3\right]} \\
=-i \frac{\beta^{2}}{2 \sqrt{2} \pi}\left(1-\frac{2 \pi}{3 \sqrt{3}}\right) \\
\text { (b) }: \frac{9 \sqrt{2} \beta^{2}}{2 p_{+}^{3}(2 \pi)^{2}} \int d^{2} k \frac{k_{+}^{3}}{\left[k^{2}-3\right]^{2}\left[(p-k)^{2}-3\right]} \\
=i \frac{\beta^{2}}{4 \sqrt{2} \pi}\left(\frac{4}{3}-\frac{7 \pi}{9 \sqrt{3}}\right) \\
\text { (c) : } i \frac{\sqrt{2}}{2}\left(1+\frac{\beta^{2}}{4 \pi}\right) Z_{1}^{\frac{1}{2}} Z_{2}^{\frac{1}{2}} \\
=i \frac{\sqrt{2}}{2}\left(1+\frac{\beta^{2}}{4 \pi}\right)\left[1+\frac{\beta^{2}}{4 \pi}\left(\frac{\pi}{9 \sqrt{3}}-\frac{1}{3}\right)+\ldots\right]
\end{gathered}
$$

The last contribution contains the $\beta^{2}$ corrections to the current, as well as one-loop wave-function renormalization factors. Therefore, up to order $\beta^{2}$ we find the spin 2 charge

$$
\omega_{ \pm}= \pm\left(\frac{1}{\sqrt{2}}+\frac{\beta^{2}}{6 \sqrt{6}}\right)
$$

Unlike the nonsimply-laced cases to be discussed presently, quantum effects do not change the classical charge ratio. 
Figure 1: Diagrams for the calculation of the charge; the wavy line indicates insertion of the current.

\subsection{The $a_{3}^{(2)}$ theory}

As in the previous case, we have one-loop contributions from the classical current, tree-level contributions from the one-loop corrections to the current, and contributions from wave-function renormalization. We note that the field $\phi_{1}$ has vanishing classical charge. From the lagrangian in eq. (3.8) we read the masses and the three-point couplings

$$
\begin{aligned}
& m_{1}^{2}=6 \quad, \quad m_{2}^{2}=2 \\
& \beta^{2} \mathcal{L}^{(3)}=-\phi_{1}^{3}+\phi_{1} \phi_{2}^{2}
\end{aligned}
$$

The relevant part of the current, for computations up to one-loop, is

$$
J^{(3)}=2 \partial \phi_{1} \partial \phi_{2} \partial^{2} \phi_{2}+\left(1+\frac{23 \beta^{2}}{24 \pi}\right)\left(\partial^{2} \phi_{2}\right)^{2}-\frac{\beta^{2}}{24 \pi}\left(\partial^{2} \phi_{1}\right)^{2}
$$

For the computation of $\omega_{1}$ we have contributions from the diagrams in Fig.1 which give

$$
\begin{gathered}
\text { (a) }: \frac{4 i \beta^{2}}{p_{+}^{4}(2 \pi)^{2}} \int d^{2} k \frac{p_{+} k_{+}^{2}(p-k)_{+}}{\left[k^{2}-2\right]\left[(p-k)^{2}-2\right]} \\
=\frac{\beta^{2}}{12 \pi}\left(1-\frac{4 \pi}{3 \sqrt{3}}\right) \\
(b): \frac{4 i \beta^{2}}{p_{+}^{4}(2 \pi)^{2}} \int d^{2} k \frac{k_{+}^{4}}{\left[k^{2}-2\right]^{2}\left[(p-k)^{2}-2\right]} \\
=-\frac{\beta^{2}}{24 \pi}\left(1-\frac{4 \pi}{3 \sqrt{3}}\right) \\
(c):-\frac{\beta^{2}}{24 \pi}
\end{gathered}
$$

Because the classical charge vanishes there is no additional contribution from wavefunction renormalization. We obtain

$$
\omega_{1}=-\frac{\beta^{2}}{18 \sqrt{3}}+O\left(\beta^{4}\right)
$$


For the computation of $\omega_{2}$ we have contributions from a similar set of diagrams

$$
\begin{gathered}
\text { (a) : } \frac{4 i \beta^{2}}{p_{+}^{4}(2 \pi)^{2}} \int d^{2} k \frac{\left(-p_{+}\right) k_{+}(p-k)_{+}^{2}}{\left[k^{2}-2\right]\left[(p-k)^{2}-6\right]} \\
=\frac{\beta^{2}}{12 \pi}(9 \ln 3+\sqrt{3} \pi-15) \\
(b): \frac{4 i \beta^{2}}{p_{+}^{4}(2 \pi)^{2}} \int d^{2} k \frac{k_{+}^{4}}{\left[k^{2}-2\right]^{2}\left[(p-k)^{2}-6\right]} \\
=-\frac{\beta^{2}}{12 \pi}\left(\frac{9}{2} \ln 3+\frac{5 \sqrt{3} \pi}{6}-\frac{19}{2}\right) \\
(c):\left(1+\frac{23 \beta^{2}}{24 \pi}\right) Z_{2} \\
=1+\beta^{2}\left(\frac{\sqrt{3}}{36}+\frac{19}{24 \pi}\right)+O\left(\beta^{4}\right)
\end{gathered}
$$

We obtain

$$
\omega_{2}=1+\frac{\beta^{2}}{24 \pi}(9 \ln 3+\sqrt{3} \pi+8)+O\left(\beta^{4}\right)
$$

One-loop corrections do not maintain the ratio of the charges, in particular the charge of particle $\phi_{1}$ is no longer zero. As we will discuss later on, this has significant implications.

\subsection{The $c_{2}^{(1)}$ theory}

For this system the masses and relevant couplings are

$$
\begin{aligned}
& m_{1}^{2}=4 \quad, \quad m_{2}^{2}=8 \\
& \beta^{2} \mathcal{L}^{(3)}=2 \sqrt{2} \phi_{1}^{2} \phi_{2}
\end{aligned}
$$

and the relevant terms of the current are

$$
J^{(3)}=6 \sqrt{2}\left(\partial \phi_{1}\right)^{2} \partial^{2} \phi_{2}-\left(4+\frac{6 \beta^{2}}{\pi}\right)\left(\partial^{2} \phi_{1}\right)^{2}+\left(2+\frac{3 \beta^{2}}{2 \pi}\right)\left(\partial^{2} \phi_{2}\right)^{2}
$$

For the computation of $\omega_{1}$ we have the contributions from Fig.1

$$
\begin{gathered}
\text { (a) }: \frac{24 i \beta^{2}}{p_{+}^{4} \pi^{2}} \int d^{2} k \frac{p_{+} k_{+}(p-k)_{+}^{2}}{\left[k^{2}-4\right]\left[(p-k)^{2}-8\right]} \\
=\frac{\beta^{2}}{\pi}(9-3 \pi) \\
\left(b_{1}\right): \quad-\frac{32 i \beta^{2}}{p_{+}^{4} \pi^{2}} \int d^{2} k \frac{k_{+}^{4}}{\left[k^{2}-4\right]^{2}\left[(p-k)^{2}-8\right]} \\
=-\frac{\beta^{2}}{\pi}\left(1+2 \ln 2-\frac{3 \pi}{4}\right) \\
\left(b_{2}\right): \quad \frac{16 i \beta^{2}}{p_{+}^{4} \pi^{2}} \int d^{2} k \frac{(p-k)_{+}^{4}}{\left[k^{2}-4\right]\left[(p-k)^{2}-8\right]^{2}} \\
=-\frac{\beta^{2}}{2 \pi}(9-4 \ln 2-2 \pi) \\
(c) \quad: \quad-4-\frac{\beta^{2}}{\pi}\left[6-\left(1-\frac{\pi}{4}\right)\right]
\end{gathered}
$$


where the last term consists of a contribution from the one-loop correction to the current and an additional contribution from the wave-function renormalization factor.

For the computation of $\omega_{2}$ we have, in a similar manner,

$$
\begin{gathered}
\text { (a) }:-\frac{12 i \beta^{2}}{p_{+}^{4} \pi^{2}} \int d^{2} k \frac{p_{+}^{2} k_{+}(p-k)_{+}}{\left[k^{2}-4\right]\left[(p-k)^{2}-4\right]} \\
=-\frac{\beta^{2}}{4 \pi}(6-3 \pi) \\
(b): \quad-\frac{32 i \beta^{2}}{p_{+}^{4} \pi^{2}} \int d^{2} k \frac{k_{+}^{4}}{\left[k^{2}-4\right]^{2}\left[(p-k)^{2}-4\right]} \\
=\frac{\beta^{2}}{8 \pi}(8-3 \pi) \\
\text { (c) }: 2+\frac{\beta^{2}}{4 \pi}(6-1)
\end{gathered}
$$

where, in the very last paranthesis, the -1 is a contribution from wave-function renormalization.

Adding all the contributions we find the one-loop corrections to the charges

$$
\begin{aligned}
& \omega_{1}=-4-\frac{3 \beta^{2}}{2 \pi}(1+\pi)+O\left(\beta^{4}\right) \\
& \omega_{2}=2+\frac{3 \beta^{2}}{4 \pi}\left(1+\frac{\pi}{2}\right)+O\left(\beta^{4}\right)
\end{aligned}
$$

We note that here again the quantum corrections do not respect the classical charge ratio.

\section{The charge-mass renormalization connection}

It has been established [8] that for the simply-laced affine Toda theories one-loop corrections do not affect the masses of the particles except for an overall rescaling. This is not the case in the nonsimply-laced theories [12]. In this section we discuss the connection between the quantum corrections to the masses and the charges in these theories. This connection is intimately related to, and can be derived from exact S-matrices satisfying bootstrap relations, but for the time being we stay at the lagrangian level.

We consider extending the charge conservation laws in eq. (4.2) to the threeparticle, on-shell vertex function. As we discuss in the next section, this extension suffers from one notable failure when applied to the $\left\langle\phi_{1} \phi_{1} \phi_{1}\right\rangle$ vertex function in the $a_{3}^{(2)}$ theory, due to the presence of an on-shell anomalous threshold singularity. Postponing discussion of this case, we recall first the consequences of momentum and spin-3 charge conservation for an on-shell vertex function $\left\langle\phi_{a} \phi_{b} \phi_{c}\right\rangle$ :

$$
\begin{aligned}
& p_{+a}+p_{+b}+p_{+c}=0 \\
& \omega_{a} p_{+a}^{3}+\omega_{b} p_{+b}^{3}+\omega_{c} p_{+c}^{3}=0
\end{aligned}
$$


with $p_{+}=\frac{m}{\sqrt{2}} e^{\theta}$ in terms of the rapidity. In particular, for the vertex function $\left\langle\phi_{a} \phi_{a} \phi_{b}\right\rangle$, in the frame of reference where the two particles $\phi_{a}$ have rapidity $\pm i \theta$ while the third particle has rapidity zero (the on-shell condition forces the momenta to be complex in general) the conservation laws read

$$
\begin{aligned}
2 m_{a} \cos \theta & =m_{b} \\
2 \omega_{a} m_{a}^{3} \cos 3 \theta & =\omega_{b} m_{b}^{3}
\end{aligned}
$$

unless the vertex function vanishes. If $a \neq b$ one derives the following relation:

$$
\frac{\omega_{b}}{\omega_{a}}=1-3 \frac{m_{a}^{2}}{m_{b}^{2}}
$$

(In particular, if $\omega_{b}=0$ either $\omega_{a}=0$ or else the mass ratio must be $\frac{m_{b}}{m_{a}}=\sqrt{3}$.)

We consider now the charge conservation laws in the $c_{2}^{(1)}$ theory, applied to the vertex function $\left\langle\phi_{1} \phi_{1} \phi_{2}\right\rangle$. At the classical level, with $\frac{m_{1}}{m_{2}}=\frac{1}{\sqrt{2}}$, the above relation implies $\frac{\omega_{2}}{\omega_{1}}=-\frac{1}{2}$ which is indeed in agreement with the values of the classical charges. We extend now these results to the one-loop level. From Ref. [12] we record the one-loop correction to the mass ratio

$$
\frac{m_{1}^{2}}{m_{2}^{2}}=\frac{1}{2}-\frac{\beta^{2}}{32}
$$

which, when substituted into eq. (5.3) gives

$$
\frac{\omega_{2}}{\omega_{1}}=-\frac{1}{2}+\frac{3 \beta^{2}}{32}
$$

which is indeed in agreement with the one-loop corrections to the charges that we have computed in eq. (4.22).

The same procedure can be used for the vertex function $\left\langle\phi_{2} \phi_{2} \phi_{1}\right\rangle$ of the $a_{3}^{(2)}$ theory. At the classical level, using the mass ratio $\frac{m_{2}}{m_{1}}=\frac{1}{\sqrt{3}}$ we conclude that either the coupling $\phi_{1} \phi_{2}^{2}$ is zero, or the charge $\omega_{1}=0$. In fact the coupling is not zero, but the classical charge vanishes. We consider now the situation at the one-loop level. Here, from Ref. [12] we have,

$$
\frac{m_{2}^{2}}{m_{1}^{2}}=\frac{1}{3}+\frac{\beta^{2}}{54 \sqrt{3}}
$$

Using eq. (5.3) we obtain

$$
\frac{\omega_{1}}{\omega_{2}}=-\frac{\beta^{2}}{18 \sqrt{3}}
$$

which is again in agreement with the one-loop corrections in eqs. (4.15), (4.17).

We consider now the vertex function for three identical particles. Setting $a=b$ in eq. (5.2), the first equation gives $\theta=\frac{\pi}{3}$ and when substituted in the second equation it implies $\omega_{a}=0$. Therefore, either the three-point function of a field $\phi$ is zero on shell, or the corresponding spin-3 charge vanishes. More generally this 
Figure 2: Illustrating the derivation of charge conservation

is the case for any charge unless its spin is $s=6 n \pm 1$ for some integer $n$. As an example, we note that in the $c_{2}^{(1)}$ theory, where the spin-3 charge of the fields is not zero, the couplings $\phi_{1}^{3}$ and $\phi_{2}^{3}$ are indeed zero. We have checked that this also holds at the one-loop level.

On the other hand, in the $a_{3}^{(2)}$ theory, following the same reasoning we would conclude that either the on-shell vertex function $\left\langle\phi_{1} \phi_{1} \phi_{1}\right\rangle$ vanishes identically, or the corresponding charge $\omega_{1}=0$. In fact, at the classical level the coupling $\phi_{1}^{3}$ is present but the classical charge vanishes so that there is no contradiction. However at the quantum level the charge $\omega_{1}$ is not zero, and therefore the conservation arguments that lead to eq. (5.2) must break down. We examine the situation in the next section.

\section{The breakdown of charge conservation}

Given a spin $s$ conserved current, the corresponding on-shell charge conservation laws can be derived by the following argument [13]: using $\partial_{-} J_{+}^{(s)}+\partial_{+} J_{-}^{(s)}=0$ we have

$$
-\int d^{2} x e^{i \epsilon_{-} x^{-}} \partial_{-} J_{+}^{(s)}(x)=\int d^{2} x e^{i \epsilon_{-} x^{-}} \partial_{+} J_{-}^{(s)}(x)=\int d^{2} x \partial_{+}\left(e^{i \epsilon_{-} x^{-}} J_{-}^{(s)}(x)\right)=0
$$

The relation

$$
\left\langle\text { out }\left|\int d^{2} x e^{i \epsilon_{-} x^{-}} \partial_{-} J_{+}^{(s)}(x)\right| i n\right\rangle=0
$$

has the graphical interpretation shown in Fig.2: one inserts the operator $\partial_{-} J_{+}^{(s)}$, carrying momentum $\epsilon=\left(0, \epsilon_{-}\right)$in all possible ways in the Feynman diagram for the process $\langle$ out $|$ in $\rangle$ and the result must be zero.

When the operator is inserted in an external line with on-shell momentum $p$ one gets a factor proportional to $\omega p_{+}^{s+1}$ from the current, a factor $\epsilon_{-}$from the derivative, and a factor with a pole singularity as $\epsilon_{-} \rightarrow 0$

$$
\frac{1}{(p+\epsilon)^{2}-m^{2}}=\frac{1}{2 p_{+} \epsilon_{-}}
$$


Figure 3: Diagrams showing the breakdown of the naive charge conservation law

from the additional propagator with on-shell external momentum. When the current is inserted into an internal line, one normally gets contributions which do not have a pole singularity in the limit $\epsilon_{-} \rightarrow 0$ but still have the numerator factor $\epsilon_{-}$. Therefore in the limit $\epsilon_{-} \rightarrow 0$ one finds $\left(\sum_{i} \omega_{i} p_{i+}^{s}\right)\langle$ out $\mid i n\rangle=0$ implying that either the sum of the charges is zero or the corresponding on-shell amplitude vanishes.

A priori this result holds also for the three-point function, although momentum conservation and the on-shell conditions require that the momenta be complex. In particular, in two dimensions, it is implied by the S-matrix bootstrap. However, precisely in two dimensions, and for example in the case of the $\left\langle\phi_{1} \phi_{1} \phi_{1}\right\rangle$ on-shell vertex function of the $a_{3}^{(2)}$ theory, the argument breaks down; contributions which come when the current is inserted inside the Feynman diagram have pole singularities as $\epsilon_{-} \rightarrow 0$ because for the particular values of the masses the diagrams represented in Fig.3b,c have anomalous threshold singularities precisely when the three external lines are on the mass-shell, as follows from standard dual diagram analysis [8, 11]. Thus, as already suggested by the contradiction we found in the previous section, the naive charge conservation breaks down, because in the limit $\epsilon_{-} \rightarrow 0$ there are additional contributions to the (integrated) current conservation law. In this section we demonstrate explicitly this phenomenon for the particular example of the $\left\langle\phi_{1} \phi_{1} \phi_{1}\right\rangle$ vertex.

At the one-loop level we need consider the set of diagrams illustrated in Fig.3, carry out the computation for $\epsilon_{-} \neq 0$, and then take the limit $\epsilon_{-} \rightarrow 0$. When the current is inserted in the external $\phi_{1}$ lines the classical part gives zero, since $\omega_{1}^{(0)}=0$ and we are left with a contribution proportional to $\omega_{1}^{(1)}\left(p_{+}^{3}+q_{+}^{3}+k_{+}^{3}\right)$ times the classical vertex function. When the current is inserted in an internal $\phi_{2}$ line, or at a vertex containing the $\phi_{2}$ field, we get a contribution from the classical part. Thus, we must compute the one-loop diagrams in Fig.3b,c representing

$$
\left\langle p, q, k\left|\int d^{2} x e^{i \epsilon_{-} x^{-}} \partial_{-}\left[2 \partial \phi_{1} \partial \phi_{2} \partial^{2} \phi_{2}+\left(\partial^{2} \phi_{2}\right)^{2}\right]\right| 0\right\rangle
$$

When $\epsilon_{-} \neq 0$ these diagrams are not at their anomalous threshold value. However, in the limit $\epsilon_{-} \rightarrow 0$ the anomalous threshold singularities become simple poles in $\epsilon_{-}$and this cancels the corresponding $\epsilon_{-}$numerator factor leading to a finite contribution to the charge conservation law.

We evaluate the Feynman integrals corresponding to the diagrams in Fig.3, with the external lines on-shell, and with the current insertion introducing a momentum 
$\epsilon=\left(0, \epsilon_{-}\right)$. Each diagram corresponds to three possible places for the current insertion. We use

$$
\begin{aligned}
& 2 p_{+} p_{-}=2 q_{+} q_{-}=2 k_{+} k_{-}=m_{1}^{2}=6 \\
& p_{+}+q_{+}+k_{+}=0 \quad, \quad p_{-}+q_{-}+k_{-}=\epsilon_{-} \\
& p_{+}^{3}+q_{+}^{3}+k_{+}^{3}=\frac{3 m_{1}^{3}}{2 \sqrt{2}}+O\left(\epsilon_{-}\right)
\end{aligned}
$$

where for the last relation we chose the rest frame of one of the particles.

The insertion of the current into the external lines leads to a contribution

$$
(a)=\frac{12 \epsilon_{-} p_{+}^{4} \omega_{1}}{(p-\epsilon)^{2}-m_{1}^{2}}+\operatorname{cyclic}(p, q, k)=-6\left(p_{+}^{3}+q_{+}^{3}+k_{+}^{3}\right) \omega_{1}
$$

If this were the only contribution, from eq. (6.2) we would conclude that $\omega_{1}$ must vanish. However, $\omega_{1}=-\frac{\beta^{2}}{18 \sqrt{3}}$, cf. eq. (4.15), and using eq. (6.5) we obtain

$$
(a)=3 \beta^{2}
$$

The diagram in Fig.3b leads to the integral

$$
(b)=\frac{8 i \beta^{2}}{(2 \pi)^{2}} \int d^{2} l \frac{\epsilon_{-} q_{+}^{2}(l+q)_{+} l_{+}}{\left[(l-k)^{2}-2\right]\left[(l+q-\epsilon)^{2}-2\right]\left[l^{2}-2\right]}+\operatorname{cyclic}(p, q, k)
$$

whereas the diagram in Fig.3c gives

$$
(c)=-\frac{16 \beta^{2}}{(2 \pi)^{2}} \int d^{2} l \frac{\epsilon_{-} l_{+}^{4}}{\left[l^{2}-2\right]\left[(l+q)^{2}-2\right]\left[(l+p+q)^{2}-2\right]\left[(l+\epsilon)^{2}-2\right]}+\operatorname{cyclic}(p, q, k)
$$

The numerator factors correspond to the derivatives in the current. The integrals can be computed using, for example, the partial fractions procedure described in Ref. [14] which reduces them to self-energy integrals. After a not-insignificant amount of algebra we find, in the limit $\epsilon_{-} \rightarrow 0$

$$
(b)=-\frac{3 \beta^{2}}{2 \sqrt{6}} m_{1}^{3}=-9 \beta^{2}
$$

and

$$
(c)=\frac{\beta^{2}}{\sqrt{6}} m_{1}^{3}=6 \beta^{2}
$$

The sum gives $(a)+(b)+(c)=0$, which is the correct consequence of eq. (6.2). Thus, the presence of the anomalous threshold singularities modifies the naive charge conservation law which would have led to the requirement $\omega_{1}=0$ or $\left\langle\phi_{1} \phi_{1} \phi_{1}\right\rangle=0$.

We may expect a similar breakdown of charge conservation to occur in other theories provided two ingredients are present: masses and couplings such that an on-shell vertex function has an anomalous threshold singularity, and terms in the current such that diagrams as in Fig.3b,c exist and give a nonzero contribution. In particular, this must be the case in many nonsimply-laced theories where, as discussed in Ref. [9], the bootstrap does not hold at all S-matrix poles. A similar problem seems to be absent in the simply-laced theories. 


\section{Conclusions}

In this paper we have studied the quantum properties of higher-spin currents of the affine Toda theories $a_{2}^{(1)}, a_{3}^{(2)}$ and $c_{2}^{(1)}$. These theories are classically integrable, i.e. currents exist which are conserved by virtue of the classical field equations. We have studied the spin-2 current for the $a_{2}^{(1)}$ and the spin-3 current for the $a_{3}^{(2)}$ and $c_{2}^{(1)}$ theories. We have shown that the conservation laws are maintained at the quantum level, although the currents have to be modified by higher-order corrections.

The existence of quantum-conserved currents for the affine Toda theories implies the existence of factorizable, elastic S-matrices. For the simply-laced cases exact S-matrices were proposed some time ago and checked to agree with those computed from the Toda lagrangians to some low orders of perturbation theory $[7,8]$. For the nonsimply-laced cases, e.g. the $a_{3}^{(2)}$ and the $c_{2}^{(1)}$ theories, the existence of quantumconserved currents was somewhat unexpected. Quantum corrections distort the classical mass spectrum in a manner that seemed incompatible with the existence of exact, factorizable S-matrices, and indeed attempts to construct them based on the usual bootstrap procedures had failed. However, stimulated by the existence of conserved currents, we were able to determine the S-matrices for the nonsimplylaced theories, by using more general constructions than hitherto attempted [9]. In particular, the realization that anomalous thresholds may modify the conservation relations for vertex functions made it clear that there are situations when the bootstrap principle has to be relaxed.

For situations where the S-matrix bootstrap is valid in the form

$$
S_{c d}(\theta)=S_{a d}\left(\theta+\bar{\theta}_{a c}^{b}\right) S_{b d}\left(\theta-\bar{\theta}_{b c}^{a}\right)
$$

(here the S-matrix element $S_{a b}(\theta)$ has a simple pole corresponding to particle $c$ ) the charge conservation follows by taking the logarithm of the above relation and inserting the Fourier expansion [8]

$$
\varphi_{a b}(\theta)=i \frac{d}{d \theta} \ln S_{a b}(\theta)=\sum_{s=0}^{\infty} e^{-s|\theta|} \varphi_{a b}^{(s)}
$$

so that eq. (7.1) gives

$$
\varphi_{c d}^{(s)}=\varphi_{a d}^{(s)} e^{-s \bar{\theta}_{a c}^{b}}+\varphi_{b d}^{(s)} e^{s \bar{\theta}_{b c}^{a}}
$$

In general the matrices $\varphi_{c d}^{(s)}$ have rank 1 and are related to the charges by

$$
\frac{\gamma_{a}^{(s)}}{\gamma_{b}^{(s)}}=\frac{\varphi_{a c}^{(s)}}{\varphi_{b c}^{(s)}}
$$

where $\gamma_{a}^{(s)}=\omega_{a} p_{a+}^{s}$. Eq. (7.3) expresses the charge conservation law in a threeparticle vertex function.

Failure of the conservation law in eq. (7.3), as in the example of Section 6, implies corresponding failure of the bootstrap. Indeed, in constructing the exact S-matrices in Ref. [9], we found that the bootstrap should not be applied at certain 
simple poles of the S-matrix because these poles, unlike the usual simple particle poles, are due to an interplay between the usual poles and anomalous threshold singularities. Obviously, such singularities modify the bootstrap conditions in the same manner they modify the charge conservation laws as discussed in Section 6 . However, although eqs. (7.1) and (7.3) may not be valid in every channel and for all vertex functions, there are usually enough valid bootstrap conditions so that eq. (7.4) does hold and expresses particle charges in terms of the exact S-matrix.

In Ref. [9] we have shown, using the explicit expressions for the S-matrices that for the $a_{2 n-1}^{(2)}$ theories the charges obtained from eq. (7.4) satisfy (using also $\left.p_{+}=\frac{m}{\sqrt{2}} e^{\theta}\right)$

$$
\frac{\gamma_{a}^{(s)}}{\gamma_{n}^{(s)}}=\frac{m_{a}^{s} \omega_{a}^{(s)}}{m_{n}^{s} \omega_{n}^{(s)}}=(-1)^{\frac{s-1}{2}} 2 \sin \frac{\pi s a}{H}
$$

with $H=2 n-1+\frac{\beta^{2}}{4 \pi}+O\left(\beta^{4}\right)$ while for the $c_{n}^{(1)}$ theories they satisfy

$$
\frac{\gamma_{a}^{(s)}}{\gamma_{n}^{(s)}}=\frac{m_{a}^{s} \omega_{a}^{(s)}}{m_{n}^{s} \omega_{n}^{(s)}}=\frac{\sin \frac{\pi s a}{H}}{\sin \frac{\pi s n}{H}}
$$

with $H=2 n+\frac{\beta^{2}}{2 \pi}+O\left(\beta^{4}\right)$. For our specific examples, using also the one-loop corrected masses in eqs. (5.6) and (5.4), this leads to

$$
\begin{aligned}
& \frac{\omega_{1}}{\omega_{2}}=-\frac{\beta^{2}}{18 \sqrt{3}} \\
& \frac{\omega_{1}}{\omega_{2}}=-2\left(1+\frac{3 \beta^{2}}{16}\right)
\end{aligned}
$$

for the $a_{3}^{(2)}$ and the $c_{2}^{(1)}$ theories respectively, which is indeed consistent with our explicit calculations.

We conclude with the following remark: the construction of quantum-conserved currents and charge operators insures the existence of factorized, elastic S-matrices for the theories we have considered. Aside from algebraic complexity, we believe the same construction holds for all simply-laced and nonsimply-laced Toda theories. In Refs. [7, 8] for the simply-laced cases, and in Ref. [9] for the nonsimply-laced cases, exact S-matrices have been determined and perturbative checks have been performed to identify them with those for specific Toda theories. To make the identification precise, it is necessary to specify the quantum form of the Toda lagrangians. In our construction of the conserved currents we have assumed that the exponentials are normal-ordered, and the Kač labels are chosen so that all one-point functions vanish. This last restriction is not crucial: it plays no role in checking current conservation, and can always be achieved by shifting the fields. However, it would seem that any other modification of the lagrangians, e.g. the addition by hand of finite mass counterterms, would spoil the current conservation and bring into question the existence of an exact S-matrix for the corresponding theory.

Acknowledgments. We thank P. Dorey and R. Sasaki for discussions. 


\section{References}

[1] A.V. Mikhailov, M.A. Olshanetsky and A.M. Perelomov, Comm. Math. Phys. 79 (1981) 473;

D. Olive and N. Turok, Nucl. Phys. B257[FS14] (1985) 277.

[2] A.B. Zamolodchikov and Al.B. Zamolodchikov, Ann. Phys. (NY) 120 (1979) 253.

[3] S. Parke, Nucl. Phys. B174 (1980) 166.

[4] P.P. Kulish and E.R. Nisimov, Theor. Math. Phys. 29 (1976) 922;

R. Flume, Phys. Lett. 62B (1976) 93; Phys. Lett. 68B (1977) 487;

J.H. Lowenstein and E.R. Speer, Comm. Math. Phys. 63 (1978) 97;

R. Sasaki and I. Yamanaka, Adv. Stud. Pure Math. 16 (1988) 271.

[5] T. Marinucci and S. Sciuto, Nucl. Phys. B156 (1979) 144;

E.R. Nisimov, Nucl. Phys. B163 (1980) 374;

I. Yamanaka and R. Sasaki, Prog. Theor. Phys. 79 (1988) 1167.

[6] A.B. Zamolodchikov, Int. J. Mod. Phys. A3 (1988) 743; Rutherford preprint RAL-89-001;

L. Palla, Nucl. Phys. B341 (1990) 714.

[7] A.E. Arinshtein, V.A. Fateev and A.B. Zamolodchikov, Phys. Lett. 87B (1979) 389 ;

J.L. Cardy and G. Mussardo, Phys. Lett. 225B (1989) 275;

P.G.O. Freund, T.R. Klassen and E. Melzer, Phys. Lett. B229 (1989) 243;

C. Destri and H.J. de Vega, Phys. Lett. 233B (1989) 336;

P. Christe and G. Mussardo, Nucl. Phys. B330 (1990) 465; Int. J. Mod. Phys. A5 (1990) 4581;

T.R. Klassen and E. Melzer, Nucl. Phys. B338 (1990) 485.

[8] H.W. Braden, E. Corrigan, P.E. Dorey and R. Sasaki, Nucl. Phys. B338 (1990) 689.

[9] G.W. Delius, M.T. Grisaru and D. Zanon, "Exact S-matrices for the nonsimplylaced affine Toda theories $a_{2 n-1}^{(2)}$ ", Phys. Lett. (to be published); "Exact Smatrices for nonsimply-laced affine Toda theories", CERN-TH.6337/91, IFUM 413/FT (1991).

[10] A.B. Zamolodchikov, Int. J. Mod. Phys. 4 (1989) 4235.

[11] G.W. Delius, M.T. Grisaru, S. Penati and D. Zanon, Phys. Lett. 256B (1991) 164; Nucl. Phys. B359 (1991) 125.

[12] M.T. Grisaru, S. Penati and D. Zanon, Phys. Lett. 253B (1991) 357.

[13] S. Weinberg, Phys. Rev. 135 (1964) 1049;

S.N. Vergeles and V.M. Gryanik, Sov. J. Nucl. Phys. 23 (1976) 704.

[14] M.T. Grisaru, S. Penati and D. Zanon, Nucl. Phys. B369 (1992) 373. 